\title{
Producción de orégano (Origanum sp) con fertilización nitrogenada en suelos arenosos de Corrientes
}

\author{
Burgos, Ángela M.'; Schroeder, María A.²; Cañete García, Marcela A. \\ ${ }^{1}$ Departamento de Producción Vegetal. Cátedra de Cultivos III. FCA. UNNE. \\ ${ }^{2}$ Departamento de Física y Química. Cátedra Química Analítica Agrícola. FCA. UNNE. \\ e-mail: burgosangela@agr.unne.edu.ar
}

\begin{abstract}
RESUMEN
La producción nacional de orégano distribuida mayoritariamente en las provincias de Mendoza y Córdoba no satisface la demanda de consumo interno. En la provincia de Corrientes, el orégano es altamente requerido por las industrias de chacinados locales. Para desarrollar el potencial de rendimiento del orégano, es necesario adecuar el manejo agronómico y especialmente el de las fertilizaciones, a las condiciones agroecológicas marginales, diferentes de la zona tradicional de cultivo en Argentina. El objetivo fue evaluar el rendimiento de orégano en suelos arenosos del noroeste de Corrientes, sometidos a diferentes dosis de fertilización con urea (108, 216 y $\left.325 \mathrm{~kg} \mathrm{ha}^{-1}\right)$ y un tratamiento testigo sin fertilizante. El efecto de la fertilización se determinó en dos momentos de muestreo y los resultados fueron analizados mediante un Anova, prueba de Tukey $(\mathrm{p}<0,05)$ y análisis de regresión para establecer la relación existente entre rendimiento y niveles de nitrógeno aplicados. Las variables estudiadas respondieron de manera directa y linear al incremento de la dosis del fertilizante, incrementando el rendimiento en peso fresco total (PFT) por hectárea en $270 \%$ y el peso seco de hojas (PSHo) por hectárea en alrededor de $200 \%$ con la dosis de $325 \mathrm{~kg} \mathrm{ha}^{-1}$ de úrea, respecto al testigo. El manejo de la fertilización permite alcanzar el rendimiento de $2000 \mathrm{~kg}$ de hojas secas. ha ${ }^{-1}$.año ${ }^{-1}$, citado como promedio a nivel nacional y posicionar a Corrientes como una zona con potencial para el cultivo de orégano dentro de un esquema de diversificación entre los pequeños productores locales.
\end{abstract}

Palabras clave: especie condimentaria, fertilización, rendimiento, urea.

\begin{abstract}
SUMMARY
Domestic production of oregano distributed mainly in the provinces of Mendoza and Córdoba does not meet domestic consumer demand. In Corrientes Province, oregano is highly required by local industries. To develop the potential yield of oregano, it is necessary to adapt crop management and especially of fertilizations, to this marginal agro-ecological conditions, different from the traditional growing area in Argentina. Argentina. The aim was to evaluate the performance of oregano in sandy soils northwest of Corrientes under different doses of urea fertilizer (108, 216 and $\left.325 \mathrm{~kg} \mathrm{ha}^{-1}\right)$ and a control treatment without fertilizer. The fertilization effect was determined in two sampling times and the results were analyzed by ANOVA, Tukey test $(\mathrm{p}<0.05)$ and regression analysis to establish the relationship between yields and levels of nitrogen applied. The variables studied respond directly and linearly to increasing dose of fertilizer, increasing performance in total fresh weight (PFT) per hectare in $270 \%$ and dry weight of leaves (PSHo) per hectare by about $200 \%$ with the dose of $325 \mathrm{~kg}$ $\mathrm{ha}^{-1}$ of urea, compared to the control. Fertilization management achieves performance of $2000 \mathrm{~kg}$ of dry leaves. ha $^{-1}$.year-1 cited as the national average, positioning Corrientes as an area with potential for cropping oregano as diversification way among small local producers.
\end{abstract}

Key words: seasoning species, fertilization, yield, urea.

\section{INTRODUCCIÓN}

El orégano es una de las hierbas aromáticas, procedentes de ambientes templados, destacado por su importancia económica a nivel mundial (Olivier, 1997). La producción global se estima en $60.000 \mathrm{TM}$

Recibido: 13/07/2015. Aceptado: 04/04/2016 año $^{-1}$ calculada sobre una superficie de 34.000 ha $y$ los principales países productores son Estados Unidos, Méjico, Turquía, Grecia, Israel y Marruecos (SAGPyA, 2010). Es una planta perenne, cuyo ciclo productivo puede extenderse hasta 8-10 años, el primer año de vegetación solamente es posible una cose- 
cha; a partir del segundo año pueden hacerse dos recolecciones anuales (Cano Ortiz y Lombardo, 2009).

Entre las plantas condimentarias introducidas en Argentina, el orégano, es una de las más importantes desde el punto de vista de la demanda interna, con posibilidades de exportación (De la Fuente et al., 2006). La superficie cultivada de orégano comprende alrededor del 80\% (1900 ha) de total cultivado con aromáticas en el país (Di Fabio, 2000); sin embargo la producción nacional de orégano no alcanza a cubrir la demanda nacional de la misma. El orégano se utiliza en la medicina natural como tónico general, digestivo, espasmolítico, carminativo, expectorante, antiséptico de las vías respiratorias y emenagogo; en forma externa actúa también como analgésico, cicatrizante y antiséptico. También es usado como condimento en forma seca y fresca, para dar sabor a salsas, adobos y aromatizar comidas varias. Desde el punto de vista culinario, es la hierba aromática de mayor demanda en el mundo. También se emplea para elaborar licores y en la industria de perfumería. Es por estas razones que actualmente la demanda se encuentra en alza en los mercados nacionales e internacionales y que este cultivo atrae la atención de pequeños y grandes productores (Cano Ortiz y Lombardo, 2009), cuya comercialización puede ampliarse al realizarse tanto en fresco como deshidratado.

En Argentina los puntos críticos del sistema de producción son la escasa superficie de la explotación promedio y la baja disponibilidad de capital, que generan una producción artesanal, con escasos medios mecánicos de cosecha, trilla y acondicionamiento, determinando menores rendimientos, mayores costos unitarios y un producto de deficiente e irregular calidad (Lenardis et al., 2006). La producción que actualmente se distribuye en las provincias de Mendoza, San Juan, Córdoba y Buenos Aires (Ringuelet y Lori, 1992), podría ampliarse al NEA mediante estudios de experimentación adaptativa.

El orégano es una especie que se adapta a diferentes tipos de suelos, siempre y cuando posean buen drenaje. Además, tolera valores de $\mathrm{pH}$ alcalinos y le es favorable una buena disponibilidad de fósforo y nitrógeno. Por lo tanto, es aconsejable la práctica de fertilización a lo largo de su ciclo de producción (Argüello et al., 2012).

La fertilización del orégano no ha sido muy estudiada en Argentina y es escasa la información existente en el mundo, solo se registran recomendaciones empíricas (Ratti, 1986; Muñoz, 1993; Arizio et al, 1996). Ringuelet et al. (2005) en ensayos realizados en un suelo Argiudol típico en La Plata (Buenos Aires), con $0,14 \%$ de $\mathrm{N}$ total concluyeron que una dosis de $\mathrm{N}$ de $80 \mathrm{~kg} \mathrm{ha}^{-1}$ provocó respuestas significativas en el incremento de los rendimientos, siendo innecesarias dosis más elevadas. Estudios más recientes permitieron confirmar que fertilizando con una dosis media de P (46 Unidades), y aumentando la dosis de fertilización nitrogenada de 18 a 156 Unidades de $\mathrm{N}$, es posible incrementar los rendimientos en forma progresiva (Suárez, 2010). Este autor destaca asimismo, la necesidad de realizar la evaluación económica de la aplicación de fertilizantes y su impacto en el rendimiento.

Dadas las condiciones agroecológicas del norte del país, el orégano es un cultivo con potencial productivo en los suelos arenosos de Corrientes, donde es altamente requerido por las industrias de chacinados locales dado que garantiza inocuidad y estabilidad de alimentos, protegiendo de alteraciones lipídicas. Para desarrollar este potencial, es necesario adecuar el manejo de las fertilizaciones a las condiciones agroecológicas del noroeste de Corrientes, con situaciones edafoecológicas diferentes de la zona tradicional de cultivo en Argentina. En este sentido, el objetivo de este trabajo fue evaluar el rendimiento de orégano en suelos arenosos del noroeste de Corrientes, sometidos a diferentes dosis de fertilización nitrogenada.

\section{MATERIALES Y MÉTODOS}

La experiencia fue llevada a cabo en el Campo Didáctico-Experimental de la Facultad de Ciencias Agrarias de la Universidad Nacional del Nordeste, ubicado en el Departamento Capital de la Provincia de Corrientes $\left(27^{\circ} 28^{\prime} 28.38^{\prime \prime} \mathrm{S}, 58^{\circ} 46^{\prime} 55.43^{\prime \prime} \mathrm{O}\right.$, 50 m.s.n.m.). El material biológico utilizado fueron plantas madres de Origanum sp. de tres años de edad, que crecían en el huerto de plantas aromáticas de la Cátedra de Cultivos III instalado en el mencionado campo experimental, de las cuales se realizaron divisiones de matas para implantar el ensayo.

El clima de la zona se caracteriza por presentar precipitaciones promedio de $1.300 \mathrm{~mm}$ anuales, evapotranspiración media anual según Thornthwaite de $1.100 \mathrm{~mm}$ y una temperatura media anual de $21,6^{\circ} \mathrm{C}$; con un período libre de heladas de 340 a 360 días. De acuerdo a los datos meteorológicos mencionados y tomando la clasificación climática de Köppen la región se clasifica como Cf w'a (h) que expresa un clima mesotermal, cálido templado, sin estación seca con precipitaciones máximas en otoño y veranos muy cálidos con temperaturas superiores a $\operatorname{los} 22^{\circ} \mathrm{C}$ y media superior a los $18^{\circ} \mathrm{C}$. Por sus características, según Köppen corresponde a Climas Templados Húmedos (De Fina y Ravelo, 1985; Bruniard, 2000).

El suelo del sitio de experimentación se clasifica como Udipsammentes árgico, familia mixta, hipertérmica, pertenece a la Serie Ensenada Grande, se caracteriza por su baja fertilidad, baja CIC pero muy buenas condiciones físicas asociadas al buen drenaje (Escobar et al., 1994). El análisis químico del sue- 
lo llevado a cabo en la Cátedra de Edafología de la Facultad de Ciencias Agrarias (UNNE) arrojó los siguientes resultados: Materia orgánica: $0,9 \%$, Nitrógeno total disponible: $0,045 \%$, Fósforo aprovechable: 65,48 ppm, K: 0,30 meq $100 \mathrm{~g}^{-1}$ suelo; $\mathrm{pH}: 6,26$.

La preparación del suelo fue realizada un mes antes del trasplante con labranza convencional y la implantación del ensayo fue realizado manualmente en el mes de noviembre de 2012, por división de matas de las plantas madres, estableciéndose una densidad de $40.000 \mathrm{pl} \mathrm{ha}^{-1}$.

El diseño experimental utilizado fue en bloques completos al azar (DBCA) con cuatro tratamientos y tres repeticiones por tratamiento. El diseño en DBCA, fue elegido a fin de intentar controlar la heterogeneidad que pudiera surgir del suelo del lote, cuya historia es la de pertenecer a un campo experimental donde numerosos ensayos han sido conducidos a través de años de experimentación y de potenciales proyecciones de sombra sobre el ensayo.

Los tratamientos consistieron en distintas dosis de urea granulada (46-0-0) distribuidos de la siguiente manera:

T1: Testigo sin fertilizar,

T2: fertilizado con $108 \mathrm{~kg}$ urea ha-1,

T3: con $217 \mathrm{~kg}$ urea ha-1 $\mathrm{y}$

T4: con $320 \mathrm{~kg}$ urea ha-1.

Cada parcela experimental consistió en $6 \mathrm{~m}^{2}$ con 3 lineos (separados a 0,50 m) de 7 plantas cada uno (a $0,50 \mathrm{~m}$ ), solo el lineo central de cada repetición se muestreó.

El fertilizante (urea granulada) fue aplicado 10 días después trasplante, se distribuyó a lo largo de cada surco, a unos $15 \mathrm{~cm}$ de distancia y a $3 \mathrm{~cm}$ de profundidad de cada planta.

Durante el ensayo, las malezas fueron controladas manualmente con azadas. En ningún momento del ensayo fue necesario recurrir a la aplicación de productos insecticidas y/o fungicidas.

Dos cosechas fueron realizadas y muestreadas, la primera en el mes de Abril de 2013 y la segunda en Octubre de 2013. El segundo corte se realizó sobre el rebrote del primero. Las cosechas fueron realizadas manualmente con tijera de podar; a unos $15 \mathrm{~cm}$ de altura del cuello de las plantas. Una vez cosechadas las muestras fueron pesadas en fresco y luego secadas en estufa a temperatura de $50{ }^{\circ} \mathrm{C}$ hasta peso constante, para evitar pérdidas por evaporación de aceite esencial y de calidad en el producto obtenido (Ringuelet y Cerimele, 1987; Ringuelet et al., 2004) y posteriormente pesadas de manera particionada en hojas y tallos.

A partir de las variables medidas en las muestras $\left(\mathrm{g} \mathrm{pl}^{-1}\right)$, se estimaron los rendimientos en $\mathrm{kg} \mathrm{ha}^{-1}$ : Peso fresco de la biomasa aérea (PFT), Peso seco de hojas (PSHo), Peso seco de tallos (PSTa), Peso seco de total
(PST). La altura máxima de plantas (AP) fue medida en cm, desde la base hasta el ápice en sentido acrópeto con regla milimetrada, tomándose el valor del tallo más alto de cada planta, el registro se realizó mensualmente desde diciembre de 2012 a la cosecha final en octubre 2013, el único mes que no se registró fue en mayo de 2013 dado que la primera cosecha se realizó en abril y se esperó que las plantas rebrotaran para poder tomar el siguiente dato.

Para analizar estadísticamente las variables fue realizado un ANOVA, comparando los promedios mediante la prueba de Tukey $(\mathrm{p}<0,05)$. También se efectuó un análisis de regresión para establecer la relación existente entre PFT y el PSHo con las dosis de urea aplicadas. Para los análisis se utilizó el software Infostat (Di Rienzo et al., 2011).

\section{RESULTADOS Y DISCUSIÓN}

En cuanto a los efectos de los tratamientos sobre las variables estudiadas, los incrementos en las dosis de nitrógeno generaron respuestas positivas en términos de rendimiento del cultivo, en coincidencia con numerosas investigaciones llevadas a cabo en otras zonas de producción de orégano (Ratti, 1986; Muñoz, 1993; Arizio et al., 1996; Ringuelet et al., 2005).

En relación al PFT de la biomasa aérea (hojas y tallos), en la primera cosecha no se encontró diferencia estadísticamente significativa entre tratamientos (Tabla 1). Sin embargo, en la segunda cosecha, el tratamiento T4, generó diferencias significativas respecto del testigo (Tabla 2), incrementando el PFT en $270 \%$, casi triplicando el rendimiento. Estos resultados mostraron una respuesta altamente favorable a la fertilización nitrogenada en el cultivo de orégano en suelos arenosos esqueléticos de la zona norte de Corrientes y serían contundentes para la comercialización del producto fresco.

En lo que se refiere al material deshidratado, solo el PSTa permaneció indiferente a los tratamientos durante el primer muestreo (Tabla 1), pero el PSHo y consecuentemente el PST mostró diferencias estadísticas durante el primer (Tabla 1) y segundo muestreo (Tabla 2). Particularmente, el PSHo respondió significativamente de manera temprana a la aplicación del fertilizante en las dos dosis más altas probadas, obteniéndose valores de $172 \mathrm{~kg} \mathrm{ha}^{-1}$ y $144 \mathrm{~kg} \mathrm{ha}^{-1}$, para los tratamiento $\mathrm{T} 3$ y $\mathrm{T} 4$ respectivamente (Tabla 1), lo que representó incrementos de $230 \%$ y $192 \%$ respecto del testigo.

El hecho de que en el primer muestreo se haya incrementado el PSHo sin haber incrementos en el PSTa, resulta sumamente conveniente para la producción y comercialización del producto deshidratado, ya que solo se admiten las hojas y sumidades florecidas, sanas, limpias y secas en su composición 
Tabla 1. Producción peso fresco total (PFT), peso seco de hojas (PSHo), peso seco de tallos (PSTa) y peso seco total (PST) por hectárea en el muestreo 1 (abril/2013) de los diferentes tratamientos con fertilización nitrogenada en Origanum vulgare, para una densidad de 40.000 plantas $_{\text {ha }}^{-1}$.

\begin{tabular}{lcccc}
\hline Tratamiento & $\begin{array}{c}\text { PFT } \\
\left(\mathrm{kg} \mathrm{ha}^{-1}\right)\end{array}$ & $\begin{array}{c}\text { PSHo } \\
\left(\mathrm{kg} \mathrm{ha}^{-1}\right)\end{array}$ & $\begin{array}{c}\text { PSTa } \\
\left(\mathrm{kg} \mathrm{ha}^{-1}\right)\end{array}$ & $\begin{array}{c}\text { PST } \\
\left(\mathrm{kg} \mathrm{ha}^{-1}\right)\end{array}$ \\
\hline T1 & $531,00 \mathrm{a}$ & $74,67 \mathrm{a}$ & $33,60 \mathrm{a}$ & $108,27 \mathrm{a}$ \\
T2 & $698,33 \mathrm{a}$ & $89,33 \mathrm{ab}$ & $45,47 \mathrm{a}$ & $134,80 \mathrm{ab}$ \\
T3 & $794,00 \mathrm{a}$ & $172,00 \mathrm{c}$ & $52,80 \mathrm{a}$ & $224,80 \mathrm{c}$ \\
T4 & $851,33 \mathrm{a}$ & $144,00 \mathrm{bc}$ & $58,67 \mathrm{a}$ & $202,67 \mathrm{bc}$ \\
\hline CV \% & 19,93 & 19,27 & 25,35 & 19,19
\end{tabular}

Medias con una letra común no son significativamente diferentes $(\mathrm{p}<=0,05)$.

Tabla 2. Producción peso fresco total (PFT), peso de hojas (PSHo), peso seco de tallos (PSTa) y peso seco total (PST) por hectárea en el muestreo 2 (octubre/ 2013) de los diferentes tratamientos con fertilización nitrogenada en Origanum vulgare, para una densidad de 40.000 plantas ha $^{-1}$.

\begin{tabular}{|c|c|c|c|c|}
\hline Tratamiento & $\begin{array}{c}\text { PFT } \\
\left(\mathrm{kg} \mathrm{ha}^{-1}\right)\end{array}$ & $\begin{array}{c}\begin{array}{c}\text { PSHo } \\
\left(\mathrm{kg} \mathrm{ha}^{-1}\right)\end{array} \\
\end{array}$ & $\begin{array}{c}\begin{array}{c}\text { PSTa } \\
\left(\mathrm{kg} \mathrm{ha}^{-1}\right)\end{array} \\
\end{array}$ & $\begin{array}{c}\text { PST } \\
\left(\mathrm{kg} \mathrm{ha}^{-1}\right)\end{array}$ \\
\hline $\mathrm{T} 1$ & $4725,71 \mathrm{a}$ & 730,47 a & $622,66 \mathrm{a}$ & 7731,42 a \\
\hline T2 & $7849,52 a b$ & $1099,04 a b$ & $723,80 a b$ & $8422,85 a$ \\
\hline T3 & $9681,90 a b$ & 1420,95 b & $1051,42 a b$ & $14125,71 a b$ \\
\hline $\mathrm{T} 4$ & $12777,14 \mathrm{~b}$ & $1542,85 \mathrm{~b}$ & $1276,19 \mathrm{~b}$ & 16104,76 b \\
\hline CV\% & 22,04 & 21,59 & 23,16 & 24,34 \\
\hline
\end{tabular}

Medias con una letra común no son significativamente diferentes $(\mathrm{p}<=0,05)$.

según lo estipulado en el capítulo XVI del Código Alimentario Argentino (De la Canal, 2002).

La respuesta del PSHo, se mantuvo en la segunda cosecha, donde se obtuvieron valores de $1.420,95 \mathrm{~kg}$ $\mathrm{ha}^{-1}$ y $1.542,85 \mathrm{~kg} \mathrm{ha}^{-1}$, en los tratamientos T3 y T4 respectivamente (Tabla 2), lo que en este caso representó incrementos de $194 \%$ y $211 \%$ respecto del T1. En esta oportunidad, el componente PSTa mostró incrementos en respuesta a las dosis crecientes de urea, con un comportamiento similar al del PSHo.

A

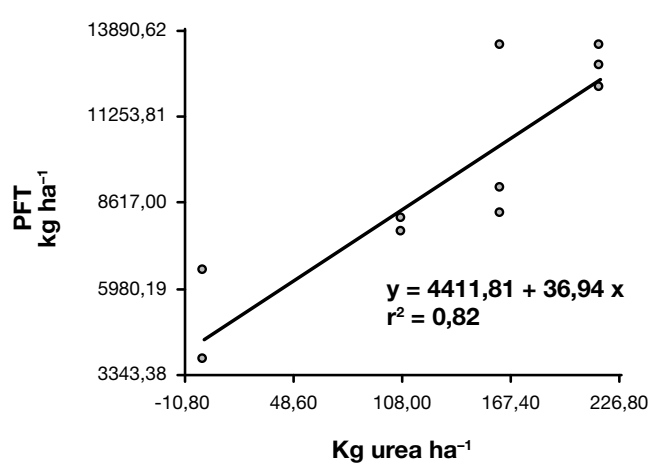

En términos de PST, en la segunda cosecha, el tratamiento T4 se diferenció significativamente de los tratamientos $\mathrm{T} 1 \mathrm{y}$ T2.

Los volúmenes de producción nacional se caracterizan por tener bajos rendimientos medios $(2000 \mathrm{~kg}$ de producto seco ha- ${ }^{-1}$ año ${ }^{-1}$ aprox.). En este ensayo se pudo obtener rendimientos de hasta $1986,85 \mathrm{~kg} \mathrm{ha}^{-1}$ año ${ }^{-1}$ al sumar las dos cosechas de hojas secas (PSHo) realizadas; fertilizando con la máxima dosis de nitrógeno ensayada (320 kg urea ha $\left.{ }^{-1}\right)$, con un stand de 40.000 plantas $\mathrm{ha}^{-1}$, lo que demuestra que es posible igualar los promedios de producción nacional. $\mathrm{Si}$ bien estos rendimientos están muy por debajo de los niveles competitivos internacionales y los potenciales de la región de mayor producción que se concentra en las provincias de Mendoza y Córdoba principalmente (4000 kg ha-1 en el primer corte) (Suárez y Ojeda, 2007), han superado ampliamente a los citados por Ringuelet et al. (2005), para zonas de producción de Provincia de Buenos Aires $\left(1.211 \mathrm{~kg} \mathrm{ha}^{-1}\right)$.

Estos resultados denotan el potencial del cultivo de orégano en Corrientes, considerada actualmente una zona marginal para el mismo y la posibilidad de contribuir a la producción nacional tanto para satisfacer la demanda interna (insatisfecha) como internacional (en crecimiento), según los citan Lenardis et al. (2006) y Argüello et al. (2012).

Las variables PFT y PSHo fueron seleccionadas por ser las determinantes del rendimiento para las dos posibles vías de comercialización de este cultivo en fresco y deshidratado, respectivamente; y a partir

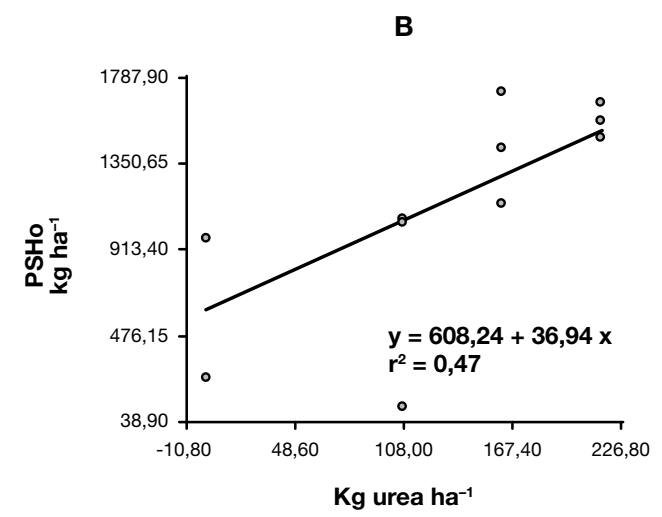

Figura 1. Relación entre la fertilización nitrogenada $\left(\mathrm{kg}\right.$ de urea ha $\left.{ }^{-1}\right)$ y el rendimiento expresado en (A) el peso fresco total (PFT) y (B) el peso seco de hojas (PSHo) en $\mathrm{kg} \mathrm{ha}^{-1}$. 
de ellas fue efectuado un análisis de regresión para establecer la relación existente entre estas variables y los niveles de urea aplicados para la segunda cosecha. En este sentido, ambas variables manifestaron una relación bastante significativa con la fertilización nitrogenada, produciendo efectos mayoritariamente favorables. El análisis de regresión mostró una respuesta lineal positiva $\mathrm{y}$ creciente, los incrementos en las dosis de urea explicaron un $82 \%$ del PFT (Fig.1 A) y un $47 \%$ (Fig.1 B) del PSHo, respectivamente. Resultados similares fueron encontrados por Ringuelet et al. (2005) en ensayos realizados en La Plata, Bs. As.

La variable biométrica cuantificada $(\mathrm{cm})$ mensualmente durante el experimento, fue la altura máxima de las plantas (AP), que mostró diferencias significativas entre tratamientos (Tabla 3). En los muestreos mensuales de 12/2012, 01/2013, $04 / 2013$ y $08 / 2013$, el tratamiento T4 se diferenció significativamente de los demás. En los meses de 02/2013, 03/2013, 06/2013, 07/2013, 09/2013 y finalmente el 10/2013 los tratamientos T3 yT4 no se diferenciaron entre ellos, pero sí respecto a los tratamientos $\mathrm{T} 1$ y T2.

La respuesta de las plantas de orégano a la fertilización nitrogenada también se manifestó en el incremento del diámetro de las plantas que fue observado durante el experimento (datos no mostrados). Luego de la segunda cosecha en octubre, se realizó una nueva división de matas de las plantas fertilizadas, y se obtuvieron entre 6 y 7 plantines por planta madre a diferencia de la primera división del que solo se lograron obtener entre 3 y 4 plantines por planta madre.

En función a estas respuestas, la fertilización nitrogenada tuvo una respuesta positiva en el rendimiento y en la tasa de multiplicación del cultivo. Estos resultados coinciden con lo expuesto por Morales y Suárez (2009) quienes reportan que el elemento más crítico para esta especie es el nitrógeno, pues en su ausencia disminuye la productividad, crecimiento y longitud de los tallos.

Similares resultados fueron encontrados por Ringuelet et al. (2005) quienes en respuesta a la fertilización nitrogenada observaron el mayor tamaño de las plantas fertilizadas y establecieron que esto se constituye en un antecedente de interés para aquellos productores que tengan como perspectiva producir plantines para su finca o para la venta.
El cultivo se presentó como altamente rústico, toleró las heladas del año 2013, no presentó plagas ni enfermedades. La competencia con malezas fue manejable y podría ser mejorada con el uso de mulchings orgánicos que contribuyan a la fertilidad edáfica.

\section{CONCLUSIONES}

El noroeste de la Pcia. de Corrientes es agroecológicamente apta para la producción del cultivo de orégano, si bien se debe optimizar el manejo agronómico para incrementar la producción del cultivo. En tal sentido, los estudios ecofisiológicos realizados hasta el momento en la región de estudio sugieren que la fertilización nitrogenada del orégano determina incrementos del $230 \%$ en el rendimiento biomasa fresca y del orden de $200 \%$ en el peso seco de hojas, con respecto al testigo, sin incrementarse el peso seco de los tallos.

Los rendimientos de orégano obtenidos en este experimento, permiten proyectarlo como un cultivo capaz de ser considerado en un plan diversificación agrícola para pequeños agricultores.

A pesar de que existe una adecuada respuesta a la fertilización por parte del cultivo, se debe optimizar el manejo de distintos ecotipos para incrementar la producción del cultivo, profundizar estudios sobre el 
manejo agronómico en términos de control de malezas, densidades de plantación y frecuencia de cortes.

\section{AGRADECIMIENTOS}

A la Secretaría General de Ciencia y Técnica de la UNNE por el financiamiento del PI A002/14.

\section{BIBLIOGRAFÍA}

Argüello, JA, Núñez, SB, Davidenco, V, Suárez, DA, Seisdedos, L, Baigorria, MC, La Porta N, Ruiz. G, Yossen, V. 2012. Sistema de producción y cadena de valor del cultivo de Orégano (Origanum sp.) en la Provincia de Córdoba (Argentina). Phyton (B. Aires) [online]. Vol.81 $\mathrm{N}^{\circ} 1$ : 23-34. ISSN 1851-5657.

Arizio, O, Curioni, A y Sánchez Vallduví, G. 1996. Economía, producción y mercado del orégano. Primeras Jornadas de Actualización en Cultivos no Convencionales Aromáticos y Medicinales. Luján, Argentina. 19 pp.

Bruniard, E. 2000. Los regímenes climáticos y la vegetación natural. Aportes para un modelo fitoclimático mundial. Academia Nacional de Geografía. p. 79. Publicación Especial No 16 . Buenos Aires, Argentina.

Cano Ortiz, A y Martínez Lombardo, MC. 2009. Cultivo de plantas medicinales en la Provincia de Jaén. Boletín del Instituto de Estudios Giennense, julio-diciembre, $\mathrm{N}^{\circ} 200$ : 195-230.

De la Canal y Asociados (Buenos Aires). Código alimentario argentino [en línea]. Buenos Aires: De la Canal, 2002. < http://www.delacanal.com.ar > Acceso exclusivo desde Biblioteca UADE. [Consulta: 10 jul. 2015].

De Fina, AL y Ravelo, AC.1985. Climatología y Fenología Agrícolas. $4^{\circ}$ Edición. EUDEBA. Buenos Aires, Argentina. $354 \mathrm{p}$.

De la Fuente E.B., Gil A, Gimenez PL, Kantolok AG, López Pereira M, Ploschuk EL, Sorlino DM, Vilariño MP, Wassner DF y. Windauer LB. 2006. Cultivos Industriales. Bs.As., Ed. Facultad de Agronomía UBA, 800 p.

Di Fabio, A. 2000. Perspectivas de producción de plantas aromáticas y medicinales en Latinoamérica. Conferencia. En: XXIII Congreso Argentino, X Latinoamericano III Iberoamericano de Horticultura. Mendoza.

Di Rienzo JA, Casanoves MG, Balzarini L, González, M, Tablada, CW y Robledo, L. InfoStat versión 2011. Grupo InfoStat FCA, Universidad Nacional de Córdoba, Argentina. URL http://www.infostat.com.ar

Escobar, EH; Ligier, D; Melgar, M; Matteio, H y Vallejos, O. 1994. Mapa de suelos de los Departamentos de Capital, San Cosme e Itatí de la Provincia de Corrientes, Argentina. p. 129. Publicación del Convenio del Instituto Nacional de Tecnología Agropecuaria (INTA-ICA) y Provincia de Corrientes-CFI, Argentina.
Lenardis, A.E; Gil, A.; Morvillo, C. 2006. “Orégano”. En: De la Fuente E.B., A. Gil, P.I. Jiménez, A.G. Kantolic, M. Lopez Pereira, E.L. Ploschuk, D.M. Sorlino, M.P. Vilariño, D.F. Warsner y L.B. Windaver (Eds.). "Cultivos Industriales”. Ed. Facultad de Agronomía, Buenos Aires.

Morales, M y Suárez, D. 2009.Evaluación de la fertilidad de un andisol en Zipaquirá (Cundinamarca, Colombia) para el cultivo de Romero (Rosmarinus officinalis) y orégano (Origanum vulgare). Trabajo de Grado. Facultad de Ciencias. Universidad Militar Nueva Granada. Colombia.118 p.

Muñoz, F. 1993. Plantas Medicinales y Aromáticas. Estudio Cultivo y Procesado. Editorial Mundi Prensa. Madrid, España. 365 pp.

Olivier, GW.1997. The World market of oregano. In: S.Padulosi (ed.), Proceedings of the IPGRI International Workshop on oregano, CIHEAM, Valenzano, Bari, Italy, pp.142- 146.España. 36.

Ratti, HA 1986. El cultivo del orégano en Catamarca. SAIPA. Publicación No 7. Buenos Aires, Argentina. p. 143146.

Ringuelet, J, Agrícola, S, Barreyro, R. 2005. Fertilización nitrogenada y rendimiento en orégano (Origanum $\mathrm{x}$ applii). Ciencia e Investigación Agraria. Vol $32 \mathrm{~N}^{\circ} 1$ : 39-43.

Ringuelet J, Cerimele, E. 1987. Secado artificial de Origanum sp., su incidencia sobre la calidad. SAIPA, 8:189194.

Ringuelet, J, Cerimele E, Henning C, Ré, MS. 2004. Influencia de la temperatura de secado en tomillo (Thymus vulgaris L.). Segundas Jornadas de Biología y Tecnología de Poscosecha. Chascomús, Buenos Aires. Resúmenes: p14.

Ringuelet, J y Lori, GA. 1992. Informe técnico sobre el cultivo de orégano en La Plata y su zona de influencia. Anales de SAIPA 9-10:27-32.

SAGPyA (Secretaria de Agricultura, Ganadería y Pesca). 2010. Orégano. http://www.sagpya.gov.ar/new/00/agricultura/otros/aromaticas/Oregano/index.php.

Suárez, D. A. y Ojeda, M. 2007. Ensayos de fertilización en orégano. Proyecto Fruti hortícola. Boletín $N^{\circ} 15$. Ed. Instituto Nacional de Tecnología Agropecuaria. 